PROCEEDINGS OF THE

AMERICAN MATHEMATICAL SOCIETY

Volume 139, Number 8, August 2011, Pages 2847-2852

S 0002-9939(2011)10754-8

Article electronically published on January 7, 2011

\title{
ON SUBSPACE-HYPERCYCLIC OPERATORS
}

\author{
CAN MINH LE \\ (Communicated by Nigel J. Kalton)
}

\begin{abstract}
In this paper we study an operator $T$ on a Banach space $E$ which is $M$-hypercyclic for some subspace $M$ of $E$. We give a sufficient condition for such an operator to be $M$-hypercyclic and use it to answer negatively two questions asked by Madore and Martínez-Avendaño. We also give a sufficient condition for $T$ to be $M$-hypercyclic for all finite co-dimensional subspaces $M$ in $E$.
\end{abstract}

\section{INTRODUCTION}

A bounded linear operator $T$ on a separable Banach space $E$ is hypercyclic if there exists a vector $x \in E$ whose orbit under $T, \operatorname{Orb}(T, x)=\left\{T^{n} x: n \geq 0\right\}$, is dense in $E$. Such a vector $x$ is called hypercyclic for $T$. Hypercyclic operators have been actively studied for more than twenty years. One can refer to [1, [2, [3] for more information about the subject.

Recently, B. F. Madore and R. A. Martínez-Avendaño in 4 introduced the concept of subspace hypercyclicity. An operator $T$ is subspace-hypercyclic for a subspace $M$ of $E$ if there exists a vector such that the intersection of its orbit and $M$ is dense in $M$. The authors prove several results analogous to the hypercyclic case. For example there is a Kitai-like criterion: if $T$ is subspace-hypercyclic, then its spectrum must intersect the unit circle; subspace-hypercyclicity is a strictly infinitedimensional phenomenon; compact or hyponormal operators are not subspacehypercyclic.

In this paper we give an improvement of the Kitai-like criterion and use it to answer negatively two questions asked by the authors in [4. Then we show that if an operator $T$ satisfies a stronger condition than the hypercyclicity criterion, it is subspace-hypercyclic for any finite co-dimensional subspace.

We use $c_{00}$ to denote the subspace of all sequences of scalars $x=(x(n))_{n=0}^{\infty}$ such that $x(0)=0$ except for finitely many $n$. If $x \in c_{00}$ let $|x|=\max \{n: x(n) \neq 0\}$.

\section{BACKWARD SHIFT OPERATOR IN $\ell_{p}$}

Let us recall some definitions from [4].

Received by the editors May 4, 2010 and, in revised form, July 28, 2010.

2010 Mathematics Subject Classification. Primary 47A16; Secondary 47B37, 37B99.

The author would like to express his thanks to Professor Richard M. Aron for his invaluable advice.

(C)2011 American Mathematical Society Reverts to public domain 28 years from publication 2847 
Definition 2.1. Let $T \in B(E)$ and $M$ be a closed subspace of $E$. We say that $T$ is $M$-hypercyclic if there exists $x \in E$ such that $\operatorname{Orb}(T, x) \cap M$ is dense in $M$. Such a vector $x$ is called an $M$-hypercyclic vector for $T$.

Definition 2.2. Let $T \in B(E)$ and $M$ be a closed subspace of $E$. We say that $T$ is $M$-transitive if for any nonempty open subsets $U, V$ of $M$ there exists a positive number $n$ such that $U \cap T^{-n} V$ contains a nonempty open subset of $M$.

In [4] the authors prove that $M$-transitivity implies $M$-hypercyclicity. Moreover, there is the following criterion.

Theorem 2.3. Let $T \in B(E)$ and $M$ be a nonzero subspace of $E$. Assume there exist $X$ and $Y$, dense subsets of $M$, and an increasing sequence of positive integers $\left(n_{k}\right)_{k=1}^{\infty}$ such that

(1) $T^{n_{k}} x \rightarrow 0$ for all $x \in X$,

(2) for each $y \in Y$, there exist a sequence $(x k)_{k=1}^{\infty}$ in $X$ such that $x_{k} \rightarrow 0$ and $T^{n_{k}} x_{k} \rightarrow y$,

(3) $M$ is an invariant subspace for $T^{n_{k}}$ for all $k$.

Then $T$ is $M$-transitive and hence $M$-hypercyclic.

Remark 2.4. The authors in [4] raise two questions relating $M$-transitivity and $M$ hypercyclicity: namely, whether conditions (1) and (2) of Theorem 2.3 are sufficient for $T$ to be $M$-hypercyclic and whether $M$-hypercyclicity implies $M$-transitivity.

In this section we answer these questions negatively by constructing counterexamples. We begin with a slight improvement of the previous theorem concerning $M$-hypercyclicity. The idea of the proof is well-known, and we include it here for the sake of completeness.

Theorem 2.5. Let $T \in B(E)$ and let $M \subset E$ be a closed subspace. Assume that there exist subsets $X$ and $Y$ of $M$ where $Y$ is dense in $M$ and an increasing sequence $\left(n_{k}\right)_{k=1}^{\infty} \subset \mathbb{N}$ such that the following hold:

(1) $T^{n_{k}}(x) \rightarrow 0$ for all $x \in X$,

(2) for each $y \in Y$ there exists a sequence $\left(x_{k}\right)_{k=1}^{\infty}$ in $X$ such that

$$
x_{k} \rightarrow 0 \text { and } T^{n_{k}} x_{k} \rightarrow y,
$$

(3) $X \subset \bigcap_{k=1}^{\infty} T^{-n_{k}}(M)$.

Then $T$ is $M$-hypercyclic.

Proof. Let $\left(\varepsilon_{j}\right)_{j=1}^{\infty}$ be a sequence of positive numbers such that

$$
\lim _{j \rightarrow \infty}\left(j \varepsilon_{j}+\sum_{i=j+1}^{\infty} \varepsilon_{i}\right)=0 .
$$

Since $E$ is separable, we can assume that $Y=\left(y_{j}\right)_{j=1}^{\infty}$ for some sequence $\left(y_{j}\right)_{j=1}^{\infty}$. We construct a sequence $\left(x_{j}\right)_{j=1}^{\infty} \subset X$ and a subsequence $\left(n_{k_{j}}\right)_{j=1}^{\infty}$ of $\left(n_{k}\right)_{k=1}^{\infty}$ by induction. Let $x_{1} \in X$ and $n_{k_{1}}$ be such that $\left\|x_{1}\right\|+\left\|T^{n_{k_{1}}} x_{1}-y_{1}\right\|<\varepsilon_{1}$. For each $j$ choose $n_{k_{j}}$ and $x_{j} \in X$ such that $\left\|x_{j}\right\|+\left\|T^{n_{k_{j}}} x_{i}\right\|+\left\|T^{n_{k_{i}}} x_{j}\right\|+\left\|T^{n_{k_{j}}} x_{j}-y_{j}\right\|<\varepsilon_{j}$ for all $i<j$. 
Let $x=\sum_{i=1}^{\infty} x_{i}$. Since $\sum_{i=1}^{\infty}\left\|x_{i}\right\| \leq \sum_{i=1}^{\infty} \varepsilon_{i}, x$ is well defined. For every $j$, we have $T^{n_{k_{j}}} x \in M$ and

$$
\begin{aligned}
\left\|T^{n_{k_{j}}} x-y_{j}\right\| & =\left\|T^{n_{k_{j}}} x_{j}-y_{j}+\sum_{i=1}^{j-1} T^{n_{k_{j}}} x_{i}+\sum_{i=j+1}^{\infty} T^{n_{k_{j}}} x_{i}\right\| \\
& \leq\left\|T^{n_{k_{j}}} x_{j}-y_{j}\right\|+\sum_{i=1}^{j-1}\left\|T^{n_{k_{j}}} x_{i}\right\|+\sum_{i=j+1}^{\infty}\left\|T^{n_{k_{j}}} x_{i}\right\| \\
& \leq j \varepsilon_{j}+\sum_{i=j+1}^{\infty} \varepsilon_{i} .
\end{aligned}
$$

Thus, $\lim _{j \rightarrow \infty}\left\|T^{n_{k_{j}}} x-y_{j}\right\|=0$ and $T$ is $M$-hypercyclic.

Remark 2.6. Notice that in Theorem 2.5 the set $X$ is not assumed to be dense in $M$. The following example shows that the first two conditions of Theorem 2.5 are not sufficient for $T$ to be $M$-hypercyclic. Thus we answer one question asked in 4 .

In $\ell_{p}$ with canonical basis $\left(e_{n}\right)_{n=1}^{\infty}$, let $B$ be the backward shift operator $B e_{n}=$ $e_{n-1}$ and let $S$ be the linear operator such that $S e_{n}=\frac{1}{2} e_{n+1}$. Let $\left(n_{k}\right)_{k=1}^{\infty}$ be an increasing sequence of positive numbers such that $n_{0}=0$ and $n_{k+1}>2 \sum_{i=0}^{k} n_{i}$. Let $L_{0}=M_{0}=\operatorname{Lin}\left\{e_{0}\right\}$ be the linear space generated by $e_{0}, L_{1}=S^{n_{1}} M_{0}$, $M_{1}=M_{0} \oplus L_{1}$, and in general let $L_{k+1}=S^{n_{k+1}} M_{k}, M_{k+1}=M_{k} \oplus L_{k+1}$. Define $M$ to be the closure of $\bigcup_{k \geq 0} M_{k}$. It is easy to show by induction that $M_{k} \subset$ $\operatorname{Lin}\left\{e_{j}: j \leq \sum_{i=0}^{k} n_{i}\right\}$ and $L_{k+1} \subset \operatorname{Lin}\left\{e_{j}: n_{k+1} \leq j \leq \sum_{i=0}^{k+1} n_{i}\right\}$. Thus, if $x \in$ $M$, then $x(j)=0$ for all $\sum_{i=0}^{k} n_{i}<j<n_{k+1}$.

Example 2.7. The operator $T=2 B$ satisfies the first two conditions of Theorem 2.5 , but $T$ is not $M$-hypercyclic.

Proof. Let $Y=\left(y_{j}\right)_{j=1}^{\infty} \subset c_{00} \cap M$ be a dense sequence in $M$. There exists an increasing sequence $\left(k_{j}\right)_{j=1}^{\infty}$ such that $y_{i} \in M_{k_{j}}$ for all $j$ and $i \leq j$. Let $X=\bigcup_{j \geq 1}\left\{S^{n_{k_{i}}} y_{j}: i \geq j\right\}$. Obviously $T$ with $X, Y,\left(n_{k_{i}}\right)_{i=1}^{\infty}$ satisfies the first two conditions of the previous theorem. It remains to show that $T$ is not $M$-hypercyclic.

Suppose that $T$ is $M$-hypercyclic with the $M$-hypercyclic vector $x \in M$. For an arbitrary $k$ there exists $m>\sum_{i=0}^{k} n_{i}$ such that $T^{m} x \in M$. One can choose $l>k$ such that $n_{l+1}-\sum_{i=0}^{l} n_{i}>2 m$. Since $\sum_{i=0}^{l} n_{i}<n_{l+1}-m<n_{l+1}-m+\sum_{i=0}^{k} n_{i}<$ $n_{l+1}$, if $n_{l+1}-m \leq j \leq n_{l+1}-m+\sum_{i=0}^{k} n_{i}$, then $\left(T^{m} x\right)(j)=0$. The fact that $\left(T^{m} x\right)(j)=2^{m} x(j+m)$ implies that $x(j)=0$ for all $n_{l+1} \leq j \leq n_{l+1}+\sum_{i=0}^{k} n_{i}$.

By the construction of $M$, it is easy to see that $M=\bigoplus_{q=0}^{\infty} S^{m_{q}} M_{l}$ for some increasing sequence $\left(m_{q}\right)_{q=0}^{\infty}$ such that $m_{0}=0$ and $m_{q+1}-m_{q}-\sum_{i=0}^{l} n_{i}>2 m$, $q \geq 0$. The same argument can be used to show that $x(j)=0$ for all $m_{q} \leq j \leq$ $m_{q}+\sum_{i=0}^{k} n_{i}, q \geq 1$. This contradicts the fact that $x$ is $M$-hypercyclic.

The following two lemmas show that $M$-hypercyclicity does not imply $M$-transitivity.

Let $\left(n_{k}\right)_{k=1}^{\infty}$ and $\left(m_{k}\right)_{k=1}^{\infty}$ be increasing sequences of positive integers such that $n_{k}<m_{k}<n_{k+1}$ for all $k$. In $\ell_{p}, p \geq 1$, consider the closed linear subspace $M$ generated by the set $\left\{e_{j}: n_{k} \leq j \leq m_{k}, k \geq 1\right\}$. 
Lemma 2.8. If $\sup _{k \geq 1}\left(m_{k}-n_{k}\right)=\infty$, then $T=2 B$ is $M$-hypercyclic.

Proof. Let $Y=\left(y_{j}\right)_{j=1}^{\infty} \subset c_{00} \cap M$ be a dense subset of $M$. Since $\sup _{k \geq 1}\left(m_{k}-n_{k}\right)=$ $\infty$, for $y_{1}$ there exist $k_{1}$ and $N_{1}$ such that $\left|y_{1}\right|<n_{k_{1}}<N_{1}<N_{1}+\left|y_{1}\right|<m_{k_{1}}$. By induction, it is easy to see that there exist increasing sequences $\left(N_{j}\right)_{j=1}^{\infty},\left(k_{j}\right)_{j=1}^{\infty}$ such that for every fixed $j>1$ we have

(1) $\left|y_{i}\right|<n_{k_{j}}<N_{j}<N_{j}+\left|y_{i}\right|<m_{k_{j}}$ for all $1 \leq i \leq j$,

(2) $N_{j}-N_{i}>n_{k_{j}}$ for all $1 \leq i<j$.

Let $X=\bigcup_{j \geq 1}\left\{S^{N_{i}} y_{j}: i \geq j\right\}$. It is clear that $X \subset M$. To verify that $T, X, Y$ and $\left(N_{j}\right)_{j=1}^{\infty}$ satisfy all conditions of Theorem 2.5 , hence that $T$ is $M$-hypercyclic, it is enough to check condition (3) that $X \subset \bigcap_{j \geq 1} T^{-N_{j}} M$.

Let $x=S^{N_{i}} y_{j} \in X$, where $i \geq j$. For every $l$ consider $T^{N_{l}} x$. If $l>i$, then $N_{l}-$ $N_{i}>n_{k_{l}}>\left|y_{j}\right|$, so $T^{N_{l}} x=T^{N_{l}-N_{i}} y_{j}=0 \in M$. If $l<i$, then $N_{i}-N_{l}+\left|y_{j}\right|<N_{i}+$ $\left|y_{j}\right|<m_{k_{i}}$ and $N_{i}-N_{l}>n_{k_{i}}$, so $T^{N_{l}} x=S^{N_{i}-N_{l}} y_{j} \in \operatorname{Lin}\left\{e_{q}: n_{k_{i}} \leq q \leq m_{k_{i}}\right\} \subset$ $M$. If $l=i$, then $T^{N_{l}} x=y_{j} \in M$.

Lemma 2.9. If $\sup _{k \geq 1}\left(n_{k+1}-m_{k}\right)=\infty$, then $T=2 B$ is not $M$-transitive.

Proof. Let $U$ and $V$ be nonempty open subsets of $M$ and suppose that there exists a positive number $m$ such that $U \cap T^{-m} V$ contains an open subset $W$ of $M$. For an element $x \in W$ there exists $\varepsilon>0$ so that if $y \in M$ and $\|x-y\|<\varepsilon$, then $y \in W$. Since $\sup _{k \geq 1}\left(n_{k+1}-m_{k}\right)=\infty$, one can find a positive number $j$ such that $n_{j+1}-m_{j}>m$. Consider $y=x+\frac{1}{2} \varepsilon e_{n_{j+1}}$. Since $y \in M$ and $\|x-y\|<\varepsilon, y \in W \subset U \cap T^{-m} V$. However, $T^{m} y=T^{m} x+\frac{1}{2} \varepsilon T^{m} e_{n_{j+1}} \in V$ implies $T^{m} e_{n_{j+1}}=2^{m} e_{n_{j+1}-m} \in M$, which contradicts the fact that $n_{j+1}-m>m_{j}$.

Proposition 2.10. If $\sup _{k \geq 1}\left(m_{k}-n_{k}\right)=\sup _{k \geq 1}\left(n_{k+1}-m_{k}\right)=\infty$, then $T=2 B$ is $M$-hypercyclic but $T$ is not $M$-transitive.

\section{Operators With DENSE GENERALIZED KERNEL}

If $T$ is a bounded linear operator in a separable Banach space $E$, then the generalized kernel of $T$ is defined to be $\operatorname{ker}^{*}(T)=\bigcup_{m=1}^{\infty} \operatorname{ker}\left(T^{m}\right)$. In this section we prove the following result.

Theorem 3.1. Let $T$ be a bounded linear operator on a separable Banach space $E$ such that $\operatorname{ker}^{*}(T)$ is dense in $E$ and there exists a map $A: \operatorname{ker}^{*}(T) \rightarrow \operatorname{ker}^{*}(T)$ satisfying

(1) $A^{m} x \rightarrow 0$ for every $x \in \operatorname{ker}^{*}(T)$,

(2) $T A=I$ on $\operatorname{ker}^{*}(T)$.

Then $T$ is $M$-hypercyclic for all finite co-dimensional subspaces $M$.

In [4] the authors raise another question concerning the relation between hypercyclicity and $M$-hypercyclicity: namely, if $T$ is hypercyclic, is $T M$-hypercyclic for some subspace $M$ ? Theorem 3.1 gives a partial answer to that question. Notice that operator $T$ satisfying the conditions of Theorem 3.1 is clearly hypercyclic. In order to prove the theorem we need the following two elementary lemmas.

Lemma 3.2. There exist a sequence of linearly independent vectors $\left(e_{p}\right)_{p=1}^{\infty}$ in $k^{*} r^{*}(T)$ such that $L=\operatorname{Lin}\left\{e_{p}, p \geq 1\right\}$ is dense in $E$, and a linear map $S: L \rightarrow L$ such that $S^{m} x \rightarrow 0$ for every $x \in L$ and $T S=I$ on $L$. 
Proof. Let $\left(x_{n}\right)_{n=1}^{\infty} \subset k e r^{*}(T)$ be a dense sequence in $E$ and $\left(e_{p}\right)_{p=1}^{\infty}$ be a maximal subset of linearly independent vectors of the set $\left\{A^{m} x_{n}, m \geq 0, n \geq 1\right\}$. If $L=$ $\operatorname{Lin}\left\{e_{p}, p \geq 1\right\}$, then $L$ is dense in $E$ and the image of $\left(e_{p}\right)_{p=1}^{\infty}$ under $A$ is in $L$. Since $\left(e_{p}\right)_{p=1}^{\infty}$ are linearly independent, we can extend $A$ linearly to $L$. The extension $S$ of $A$ satisfies all requirements of the lemma.

Lemma 3.3. If $\left(f_{j}\right)_{j=1}^{n} \subset E^{*}$, then $\bigcap_{j=1}^{n}\left(L \cap \operatorname{ker}\left(f_{j}\right)\right)$ is dense in $\bigcap_{j=1}^{n} \operatorname{ker}\left(f_{j}\right)$.

Proof. Since $L$ is dense in $E$, if $x \in \bigcap_{j=1}^{n} \operatorname{ker}\left(f_{j}\right)$, then there exists a sequence $\left(x_{k}\right)_{k=1}^{\infty} \subset L$ such that $x=\sum_{k=1}^{\infty} x_{k}$. Consider the subspace

$$
H=\operatorname{Lin}\left\{\left(f_{1}\left(x_{k}\right), f_{2}\left(x_{k}\right), \ldots, f_{n}\left(x_{k}\right)\right), k \geq 1\right\} \subset \mathbb{R}^{n} .
$$

If $\left(x_{k}\right)_{k=1}^{\infty} \subset \bigcap_{j=1}^{n} \operatorname{ker}\left(f_{j}\right)$, then there is nothing to prove. If $\left(x_{k}\right)_{k=1}^{\infty} \not \subset \bigcap_{j=1}^{n} k \operatorname{ker}\left(f_{j}\right)$, then $d=\operatorname{dim} H>0$. There exist $\left(k_{i}\right)_{i=1}^{d}$ so that $y_{i}:=\left(f_{1}\left(x_{k_{i}}\right), f_{2}\left(x_{k_{i}}\right), \ldots, f_{n}\left(x_{k_{i}}\right)\right)$, $1 \leq i \leq d$, is a basis of $H$. Let

$$
u_{m}=\left(f_{1}\left(\sum_{k=1}^{m} x_{k}\right), \ldots, f_{n}\left(\sum_{k=1}^{m} x_{k}\right)\right) \in \mathbb{R}^{n} .
$$

For every $m$ there exist scalars $\left(t_{m, i}\right)_{i=1}^{d}$ so that $\sum_{i=1}^{d} t_{m, i} y_{i}=u_{m}$. Since $u_{m} \rightarrow 0$, it follows that $t_{m, i} \rightarrow 0$ as $m \rightarrow \infty$ for all $i \leq d$. Therefore $x$ can be approximated by $\sum_{k=1}^{m} x_{k}-\sum_{i=1}^{d} t_{m, i} x_{k_{i}} \in \bigcap_{j=1}^{n}\left(L \cap \operatorname{ker}\left(f_{j}\right)\right)$.

Proof of Theorem 3.1. For simplicity of notation we prove the case when $M$ is a one-codimensional subspace of $E$. It will be clear that the argument still works for

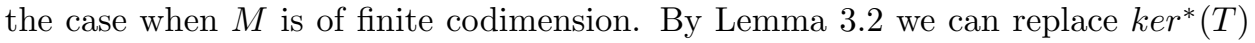
and $A$ with $L$ and $S$ respectively.

Let $M=\operatorname{ker}(f)$ for some $f \in E^{*}$. By Lemma 3.3 there exists a dense sequence $\left(x_{k}\right)_{k=1}^{\infty} \subset L$ in $M$. We construct by induction sequences $\left(y_{k}\right)_{k=1}^{\infty} \subset M$ and $\left(m_{k}\right)_{k=1}^{\infty} \subset \mathbb{N}$ with the following properties:

(1) $T^{m_{j}} y_{k} \in M$ for all $j<k$ and $T^{m_{j}} y_{k}=0$ for all $j>k$,

(2) $T^{m_{k}} y_{k}=x_{k}$,

(3) $\left\|y_{k}\right\|<2^{-k}$ and $\left\|T^{m_{j}} y_{k}\right\|<2^{-k}$ for all $j<k$.

If such sequences exist, then the same argument as in the proof of Theorem 2.5 can be used to show that $T$ is $M$-hypercyclic with the $M$-hypercyclic vector $\sum_{k=1}^{\infty} y_{k}$.

For $k=1$ consider the subspace $L_{1}=\operatorname{Lin}\left\{f\left(e_{p}\right), p \geq 1\right\}$ of $\mathbb{R}$. If $\operatorname{dim} L_{1}=0$, then $S^{m} x_{1} \in M$ for all $m \geq 1$. Since $S^{m} x_{1} \rightarrow 0$, choose $m_{1}$ large enough so that $\left\|S^{m} x_{1}\right\|<2^{-1}$ and put $y_{1}=S^{m} x_{1}$. If $\operatorname{dim} L_{1}=1$, there exists $p_{1,1} \geq 1$ so that $f\left(e_{p_{1,1}}\right) \neq 0$. Since $e_{p_{1,1}} \in L$ and $S^{m} x_{1} \rightarrow 0$, one can choose $m_{1}$ large enough so that $T^{m_{1}} e_{p_{1,1}}=0$ and $\left\|t_{1,1} e_{p_{1,1}}+S^{m_{1}} x_{1}\right\|<2^{-1}$, where $t_{1,1}=-\frac{f\left(S^{m_{1}} x_{1}\right)}{f\left(e_{p_{1,1}}\right)}$. Let $y_{1}=t_{1,1} e_{p_{1,1}}+S^{m_{1}} x_{1}$. In either case $y_{1} \in M$ by the choice of $t_{1,1},\left\|y_{1}\right\|<2^{-1}$ and $T^{m_{1}} y_{1}=x_{1}$. Thus, conditions (1), (2), and (3) are satisfied.

Suppose that we have constructed $\left(y_{k}\right)_{k=1}^{n-1} \subset M$ satisfying (1), (2), and (3). Consider the subspace $L_{n}=\operatorname{Lin}\left\{\left(f\left(e_{p}\right), f\left(T^{m_{1}} e_{p}\right), \ldots, f\left(T^{m_{n-1}} e_{p}\right)\right), p \geq 1\right\}$ of $\mathbb{R}^{n}$ and let $d=\operatorname{dim} L_{n}$. If $d=0$, then $T^{m_{j}} S^{l} x_{n} \in M$ for all $j<n$ and $l \geq 1$. Since $\left(y_{k}\right)_{k=1}^{n-1} \subset M$ and $S^{m} x_{n} \rightarrow 0$ as $m \rightarrow \infty$, one can choose $m_{n}$ large enough so that $y_{n}=S^{m_{n}} x_{n}$ satisfies conditions (1), (2), and (3). If $d \neq 0$, then there exist $p_{n, 1}, \ldots, p_{n, d}$ so that $g_{n, i}:=\left(f\left(e_{p_{n, i}}\right), f\left(T^{m_{1}} e_{p_{n, i}}\right), \ldots, f\left(T^{m_{n-1}} e_{p_{n, i}}\right)\right), 1 \leq i \leq d$, is a 
basis of $L_{n}$. Since $S^{m} x_{n} \in L, u_{n, m}=\left(f\left(S^{m} x_{n}\right), f\left(T^{m_{1}} S^{m} x_{n}\right), \ldots, f\left(T^{m_{n-1}} S^{m} x_{n}\right)\right)$ $\in L_{n}$ for all $m$. Moreover, $u_{n, m} \rightarrow 0$ as $m \rightarrow 0$. Therefore one can find $m_{n}$ large enough and scalars $\left(t_{n, i}\right)_{i=1}^{d}$ so that

(a) $u_{n, m_{n}}+\sum_{i=1}^{d} t_{n, i} g_{n, i}=0$ and $T^{m_{n}} y_{j}=0$ for all $j<n$,

(b) $T^{m_{n}} e_{p_{n, i}}=0$ for all $i \leq d$,

(c) $\left\|y_{n}\right\|<2^{-n}$ and $\left\|T^{m_{j}} y_{n}\right\|<2^{-n}$ for all $j<n$,

where $y_{n}=S^{m_{n}} x_{n}+\sum_{i=1}^{d} t_{n, i} e_{p_{n, i}}$. The properties (a), (b), and (c) ensure that $\left(y_{k}\right)_{k=1}^{n}$ satisfy conditions (1), (2), and (3). The theorem is proved.

Remark 3.4. It is easy to see that the theorem is still true if $E$ is an $F$-space or the scalar field is complex.

\section{REFERENCES}

1. F. Bayart and É. Matheron. Dynamics of linear operators, Cambridge University Press, 2009. MR2533318

2. G. Godefroy and J. H. Shapiro. Operators with dense, invariant, cyclic vector manifolds, J. Funct. Anal., 98: 229-269, 1991. MR1111569 (92d:47029)

3. K.-G. Grosse-Erdmann. Universal families and hypercyclic operators, Bull. Amer. Math. Soc. (N.S.), 36(3):345-381, 1999. MR1685272 (2000c:47001)

4. B. F. Madore and R. A. Martínez-Avendaño. Subspace hypercyclicity, J. Math. Anal. Appl. 373(2): 502-511, 2011.

Department of Mathematics and Sciences, Kent State University, Kent, Ohio 44242

E-mail address: cle@math.kent.edu 